Draft Version November 11, 2018

Preprint typeset using $\mathrm{LAT}_{\mathrm{E} X} \mathrm{X}$ style emulateapj v. 6/22/04

\title{
BLACK HOLE MASS AND BULGE LUMINOSITY FOR LOW-MASS BLACK HOLES
}

\author{
Yan-Fei Jiang ${ }^{1}$, Jenny E. Greene ${ }^{1}$ and Luis C. Ho ${ }^{2}$ \\ Draft version November 11, 2018
}

\begin{abstract}
We study the scaling between bulge magnitude and central black hole (BH) mass in galaxies with virial BH masses $\lesssim 10^{6} M_{\odot}$. Based on careful image decomposition of a snapshot Hubble Space Telescope I-band survey, we found that these BHs are found predominantly in galaxies with pseudobulges. Here we show that the $M_{\mathrm{BH}}-L_{\text {bulge }}$ relation for the pseudobulges at low mass is significantly different from classical bulges with $\mathrm{BH}$ masses $\geq 10^{7} M_{\odot}$. Specfically, bulges span a much wider range of bulge luminosity, and on average the luminosity is larger, at fixed $M_{\mathrm{BH}}$. The trend holds both for the active galaxies from Bentz et al. and the inactive sample of Gültekin et al. and cannot be explained by differences in stellar populations, as it persists when we use dynamical bulge masses. Put another way, the ratio between bulge and $\mathrm{BH}$ mass is much larger than $\sim 1000$ for our sample. This is consistent with recent suggestions that $M_{\mathrm{BH}}$ does not scale with the pseudobulge luminosity. The low-mass scaling relations appear to flatten, consistent with predictions from Volonteri \& Natarajan for massive seed BHs.

Subject headings: galaxies: active — galaxies: nuclei — methods: observational
\end{abstract}

\section{INTRODUCTION}

For massive elliptical galaxies and galaxies with classical bulges, black hole $(\mathrm{BH})$ masses have been found to correlate with various properties of bulge, such as bulge mass (e.g., Magorrian et al. 1998), bulge luminosity (the $M_{\mathrm{BH}}-L_{\text {bulge }}$ relation, see Marconi \& Hunt 2003), and the velocity dispersion of stars in the bulge (the $M_{\mathrm{BH}}-\sigma_{*}$ relation, see Tremaine et al. 2002). This has led to theoretical work exploring the importance of AGN feedback on galaxy evolution and the creation of the scaling relations (e.g., Ciotti \& Ostriker 2007). However, we still do not understand the origin of the scaling relations. Observationally, they are best-measured using nearby elliptical galaxies with central supermassive $\mathrm{BHs}$ having masses $\gtrsim 10^{7} M_{\odot}$. It is still unclear what the scaling relations look like for BHs with masses $\sim 10^{5}-10^{6} M_{\odot}$ in late-type galaxies. Some simulations (e.g., Volonteri \& Natarajan 2009) show that the $M_{\mathrm{BH}}-\sigma_{*}$ relation can result from the the hierarchical merging of massive dark matter halos (also see Peng 2007). In that picture, and assuming massive $\mathrm{BH}$ seeds $\left(\sim 10^{5} M_{\odot}\right)$, the $M_{\mathrm{BH}}-\sigma_{*}$ relation would flatten at the low-mass end. Measurement of the scaling relations of low-mass BHs will directly constrain models of $\mathrm{BH}$ seed formation and help elucidate the most important factors in establishing the observed scaling relations.

Based on Hubble Space Telescope (HST) observations of the sample of 18 low-mass BHs selected from the first data release (DR1) of the Sloan Digital Sky Survey (SDSS; Greene \& Ho 2004), Greene et al. (2008) study the $M_{\mathrm{BH}}-L_{\text {bulge }}$ relation at low mass and find a different scaling, with BHs living in larger bulges than expected from the high-mass relations. However, their sample is small and the result is quite uncertain. Greene \& Ho (2007) present 174 low-mass BHs from DR4 of the SDSS,

\footnotetext{
1 Department of Astrophysical Sciences, Princeton University, Princeton, NJ 08544, USA

2 The Observatories of the Carnegie Institution for Science, 813 Santa Barbara Street, Pasadena, CA 91101, USA
}

giving us the opportunity to study the scaling relations with an order of magnitude more objects. Their $M_{\mathrm{BH}}-\sigma_{*}$ relation has been examined by Xiao et al. (2011). We have done detailed image decompositions of these galaxies based on HST imaging (Jiang et al. 2011, hereafter Paper I) and the $M_{\mathrm{BH}}-L_{\text {bulge }}$ relation will be studied here.

Low-mass BHs are found in different host galaxy types than more massive BHs. Most of their host galaxies actually contain pseudobulges. Pseudobulges have quite different properties from those of violently and rapidly formed classical bulges. For example, pseudobulges have flatter shapes than those of classical bulges (e.g., Sérsic index $n<2$ ). They have smaller velocity dispersions at fixed luminosity in the FaberJackson relation (Faber \& Jackson 1976) compared with classical bulges (e.g., Kormendy \& Illingworth 1983; Kormendy \& Kennicutt 2004; Paper I). Pseudobulges are believed to form via secular processes in disk galaxies (see the review by Kormendy \& Kennicutt 2004). Considering their differing formation mechanisms and properties, we may expect differing $\mathrm{BH}$ scaling relations as well, which is observed with small samples with dynamical BH masses (e.g., Hu 2008, 2009; Greene et al. 2010) and indirect analysis (e.g., Gadotti \& Kauffmann 2009). As the bulges hosting the low-mass BHs presented here are likely dominated by pseudobulges (Paper I), studying the properties of these galaxies will also help us understand the growth and evolution of pseudobulges.

\section{THE SAMPLE AND DATA ANALYSIS}

The sample and observations are described in Greene \& Ho (2007) and Paper I. Here we briefly describe the key properties of the sample for completeness. The 174 galaxies are selected from DR4 of the SDSS (Greene \& Ho 2007) to have virial BH masses $<2 \times 10^{6} M_{\odot}$. They have a median redshift of 0.085 and a median $M_{\mathrm{BH}}$ of $1.2 \times 10^{6} M_{\odot}$.

The host galaxy structures and luminosities are derived from a snapshot survey with Wide Field Planetary Cam- 
era 2 (WFPC2) on HST in Cycle 16. With the Planetary Camera (PC), we achieve a resolution of $0 . \prime 246$ per pixel and a field of view of $366^{\prime \prime} 8 \times 366^{\prime \prime} 8$. The observations are done with the F814W filter (mean wavelength of $8269 \AA$ ), which we refer to as the $I$-band in the following. For each object we obtain a short $(30 \mathrm{sec})$ exposure in case of saturation, followed by two dithered $\sim 600 \mathrm{sec}$ exposures. Images are reduced following standard procedures, with extra care taken to remove cosmic ray trails (see Paper I for details).

\subsection{Black Hole Mass}

For massive BHs in the nearby universe, the most reliable $\mathrm{BH}$ masses are based on dynamical tracers such as stars (e.g., Gebhardt 2004), gas (e.g., Barth et al. 2001), or water masers (e.g., Herrnstein et al. 2005; Kuo et al. 2011). However, current instrumentation lacks the spatial resolution to obtain dynamical measurements for such low-mass BHs outside the Local Group. Thus, we use virial $\mathrm{BH}$ masses measured from active galactic nucleus (AGN) emission lines. Reverberation mapping (Blandford \& McKee 1982) provides sizes for the broadline regions (BLRs) of a few dozen AGNs, from which a relation between BLR size and AGN luminosity is calibrated (e.g., Kaspi et al. 2000; Bentz et al. 2006, 2009b). Then the virial mass is simply $M_{\mathrm{BH}}=f R(\Delta v)^{2} / G$, where $f$ is a dimensionless factor that accounts for the unknown geometry of the BLR, $R$ is the size of BLR and $\Delta v$ is some measure of the width of the broad-line width such as full width at half maximum (FWHM). In practice, the width and luminosity of the broad $\mathrm{H} \alpha$ emission line (Xiao et al. 2011) are used to estimate the virial BH masses (e.g., Greene \& Ho 2006).

Despite the large uncertainty in virial masses, it has been demonstrated that the virial masses of SMBHs are in good agreement with masses determined based on the $M_{\mathrm{BH}}-\sigma_{*}$ relation (e.g., Gebhardt et al. 2000; Ferrarese et al. 2001; Nelson et al. 2004; Greene \& Ho 2009). The uncertainty in the virial masses is thought to be a factor of $\sim 3$ (e.g., Greene \& Ho 2006; Collin et al. 2006; Shen et al. 2008; Woo et al. 2010). We adopt a single value of $f=0.75$, which is intended to represent an ensemble average over our sample and to be consistent with other people's work (e.g., Kaspi et al. 2000; Xiao et al. 2011).

\subsection{GALFIT and Bulge Luminosity}

We use detailed image decomposition to isolate the bulge component in the $I$-band images. Following Greene et al. (2008), we perform full two-dimensional fitting with GALFIT (e.g., Peng et al. 2002, 2010). Details about the fitting and the best-fitting models for our sample are given in Paper I. Here we briefly describe the basic elements.

A point-spread function (PSF) model from TinyTim (Krist 1995) is used to model the AGN component. For other components of the host galaxies, we use a generalized Sérsic (Sérsic 1968) model to fit the light. We fix the Sérsic index $n$ to be $0.5,1,2,3,4$ so that GALFIT will not converge on an unreasonably high $n$ value. If necessary, a bar is also included as an $n=0.5$ Sérsic component. The sky is fitted using a variety of methods, in order to estimate parameter uncertainties due to the sky level. Also, we use alternate PSF models to bracket
TABLE 1

FITTING RESULT

\begin{tabular}{|c|c|c|c|c|}
\hline \multicolumn{5}{|c|}{ Fitting of the $M_{\mathrm{BH}}-L_{\text {bulge }}$ relation } \\
\hline Sample & $\alpha$ & $\beta$ & $\epsilon_{0}$ & $M_{0}$ \\
\hline Bentz et al. & $-0.28 \pm 0.03$ & $8.30 \pm 0.05$ & 0.22 & -22.45 \\
\hline Bentz et al. + our sample & $-0.43 \pm 0.03$ & $6.65 \pm 0.05$ & 0.41 & -19.79 \\
\hline Gültekin et al. & $-0.46 \pm 0.05$ & $8.30 \pm 0.07$ & 0.38 & -22.55 \\
\hline Gültekin et al. + our sample & $-0.52 \pm 0.03$ & $6.41 \pm 0.05$ & 0.48 & -19.84 \\
\hline Gültekin et al. + Kormendy et al. & $-0.46 \pm 0.05$ & $8.08 \pm 0.08$ & 0.50 & -22.15 \\
\hline \multicolumn{5}{|c|}{ Fitting for $M_{\mathrm{BH}}-M_{\text {bulge }}$ relation } \\
\hline Sample & $\alpha$ & $\beta$ & $\epsilon_{0}$ & $M_{\mathrm{dyn}, 0}$ \\
\hline Gültekin et al. & $1.04 \pm 0.16$ & $8.39 \pm 0.45$ & 0.45 & 11.04 \\
\hline Gültekin et al. + our sample & $1.37 \pm 0.09$ & $6.35 \pm 0.10$ & 0.42 & 9.63 \\
\hline
\end{tabular}

the uncertainties due to errors in the PSF model. The values of the bulge luminosities and uncertainties for our sample are given in Table 2 of Paper I.

\subsection{Properties of our sample from Jiang et al. (2011)}

In Paper 1 we present evidence that the majority of the disk galaxies in our sample likely contain pseudobulges, as summarized here. Of the galaxies with reliable Sérsic index $n$ measurements, $70 \%$ have $n<2$. Extended disks are found in $92 \%$ of galaxies, while bar structures are identified in $40 \%$ of the sample. About half of the galaxies have bulge-to-total ratio $B / T$ smaller than 0.2 and $74 \%$ have $B / T<0.4$. All of our disk galaxies satisfy the relations for pseudobulges given by Gadotti (2009). Based on these properties, and their fundamental plane scalings, we argue that the disk galaxies predominantly have pseudobulges, and that the galaxies without disks are bright spheroidal, rather than elliptical, galaxies (Kormendy et al. 2009). Most striking is the FaberJackson relation. At a fixed bulge magnitude, our galaxies have smaller velocity dispersions on average.

\section{RESULTS}

We investigate the scaling relations between $\mathrm{BH}$ mass and bulge properties for our sample. Bear in mind that the absolute positions of our objects in the $M_{\mathrm{BH}}-L_{\text {bulge }}$ plane are uncertain due to potential systematic offsets in the value of $f$ and other systematic uncertainties associated with the $\mathrm{BH}$ mass measurements, as well as the unknown mass-to-light ratios of the bulges. We will discuss how each of these uncertainties may impact our conclusions.

\subsection{Black hole mass vs. bulge luminosity}

BH masses and absolute bulge magnitudes for our sample are shown in Figure $1 a$ as blue open circles. We compare our galaxies with the $M_{\mathrm{BH}}-L_{\text {bulge }}$ relations published by Bentz et al. (2009a) for reverberation-mapped AGNs and Gültekin et al. (2009) for local inactive galaxies with dynamical $\mathrm{BH}$ masses.

Bentz et al. (2009a) presents the $M_{\mathrm{BH}}-L_{\text {bulge }}$ relation for nearby $\mathrm{AGNs}^{3}$ with reverberation-based $\mathrm{BH}$ mass measurements (Figure 1 $a$ ). The bulge luminosities are

\footnotetext{
${ }^{3}$ Bright, nearby galaxies are excluded in their analysis
} 


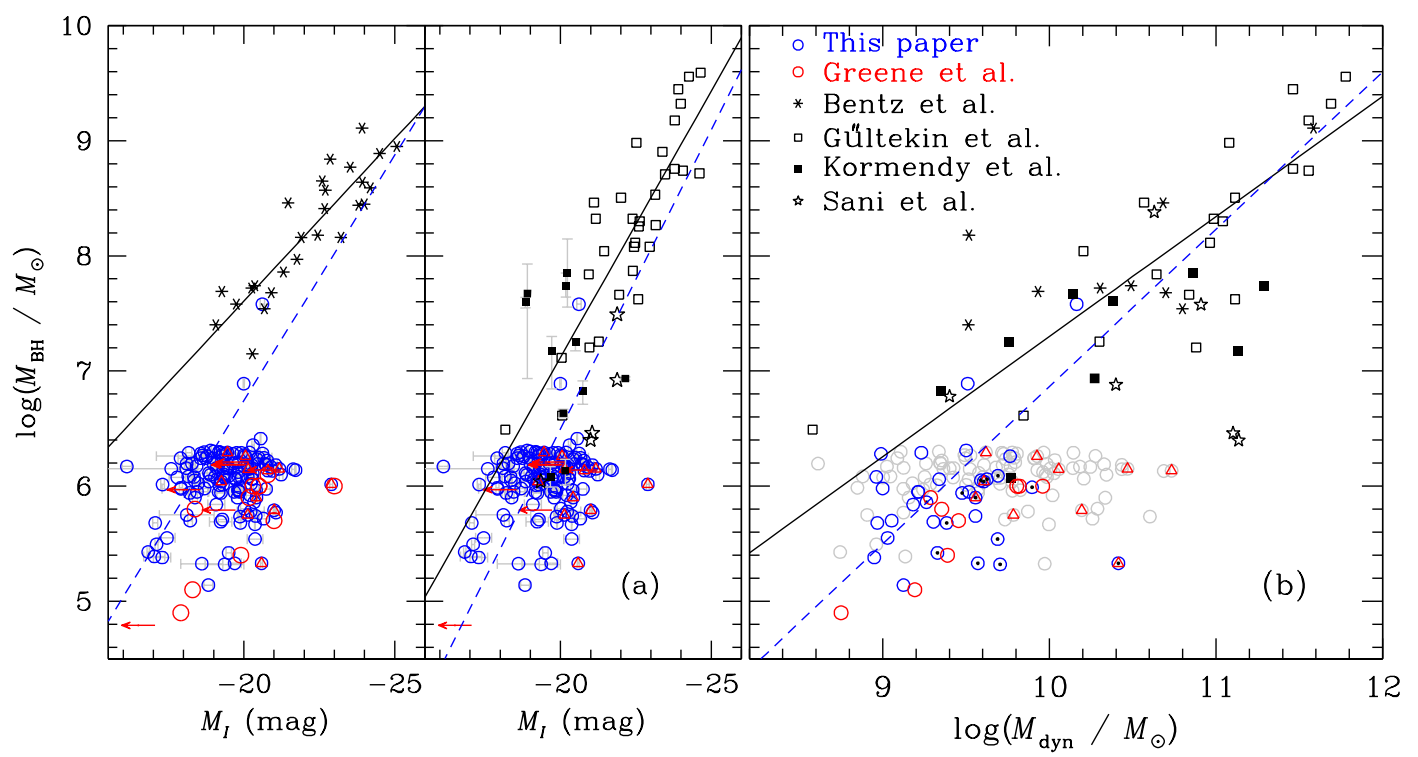

FIG. 1.-(a): The relation between the BH masses $M_{\mathrm{BH}}$ and $I$-band absolute magnitude $M_{I}$ of the bulge. Our sample is labeled with open blue circles. Galaxies without an extended disk are labeled with red triangles. Upper limits of the bulges for bulgeless galaxies are plotted with arrows. Solid lines are the best fitting relation for active galaxies from Bentz et al. (2009a) and inactive galaxies from Gültekin et al. (2009), respectively, in the two sub-panels. Dashed blue lines are the best-fitting relations when our sample is included. All comparison samples are shifted to the $I$-band appropriately. (b): The relation between $\mathrm{BH}$ mass and dynamical bulge mass. Grey open circles are objects from our sample with velocity dispersions measured from [S II] (Greene \& Ho 2007). Open blue circles with black dot at the center are galaxies with little disk contamination for the stellar velocity dispersion measurements. We show the best-fit relation of the Gültekin et al. sample using dynamical bulge masses from Häring \& Rix (2004) (solid line) and the best fit including the subsample of galaxies for which we have stellar velocity dispersions (blue line) .

based on GALFIT modeling of HST/F550M or F547M images. Since our virial BH masses are based on a radiusluminosity relation derived from these same objects, the $\mathrm{BH}$ masses are directly comparable, hopefully minimizing systematic errors. However, we note that their $\mathrm{BH}$ masses range from $10^{7}-10^{9} M_{\odot}$, larger than the $\mathrm{BH}$ masses in our sample by about 2 orders of magnitude. This comparison can tell us whether the $M_{\mathrm{BH}}-L_{\text {bulge }}$ relation that is observed in massive AGNs still exists at the low masses probed here.

In order to make this comparison, we adopt the $f$ factor from Onken et al. (2004, e.g., we boost our virial BH masses by $0.3 \mathrm{dex}$ ). We fit the sample with the following log-linear relation

$$
\log \left(M_{\mathrm{BH}} / M_{\odot}\right)=\alpha\left(M_{I}-M_{0}\right)+\beta,
$$

where $M_{0}$ is the median magnitude measured from the fitted sample, which is held constant during the fitting. For the following fits, we do not include galaxies with only upper limits on their bulge magnitudes ${ }^{4}$. Following Greene \& Ho (2006) and Tremaine et al. (2002), we include the intrinsic scatter $\epsilon_{0}$ in the definition of $\chi^{2}$ (Equation 1 of Greene \& Ho 2006) so that the best fit gives a $\chi^{2}=1$. Thus, we get an upper limit on the intrinsic scatter.

Compared to the Bentz et al. fit, we find a significantly steeper slope and lower zeropoint when our sample is included. The difference between the two samples can be quantified by calculating the predicted $\mathrm{BH}$ masses

\footnotetext{
${ }^{4}$ We have tried to fit our sample including the upper limits using the maximum likelihood method as done in Gültekin et al. (2009). The answer is unchanged.
}

$M_{\text {predict }}$ based on our bulge luminosities and the bestfitting relation for the Bentz et al. sample. We calculate a mean difference of $\left\langle\log \left(M_{\text {predict }} / M_{\mathrm{BH}}\right)\right\rangle=1.15 \pm 0.03$, where the latter is the error on the mean. When we fit our combined samples, we find an intrinsic scatter of $\epsilon_{0}=0.41-0.45$ for an assumed scatter in virial BH masses of 0.48 (e.g., Collin et al. 2006; Woo et al. 2010) to 0.4 (e.g., Greene \& Ho 2006). Both calculations show that the galaxies in our sample have systematically smaller BH masses at a fixed bulge mass compared with the sample of Bentz et al. Differences in stellar populations, which we estimate would dim our bulges by $\sim 1$ mag (Paper I), are not sufficient to explain these discrepancies.

To investigate further whether these differences represent a real physical difference (e.g., as a function of mass), we also compare to inactive $\mathrm{BHs}$ with dynamical BH masses from Gültekin et al. (2009). As explained in Paper I, we adopt a color difference of $V-I=1.34$ mag to shift the Gültekin et al. sample from the $V$-band to the $I$-band. The average difference between the predicted and observed $\mathrm{BH}$ masses based on the Gültekin et al. fit is $\left\langle\log \left(M_{\text {predict }} / M_{\mathrm{BH}}\right)\right\rangle=0.85 \pm 0.04$. If we fit our sample combined with that of Gültekin et al., the intrinsic scatter increases significantly (see Table 1). This scatter is driven by a systematic shift between our sample and the Gültekin et al. sample. For a given BH mass, our galaxies have larger bulge luminosities. This shift may be real, or it may be explained by younger stars, and thus lower mass-to-light ratios, in our sample galaxies (Paper I). In order to investigate the magnitude of this uncertainty, we estimate dynamical masses $M_{\mathrm{dyn}}$ 
for a subset of our sample, as described below. Dynamical mass should be a good approximation of the stellar mass of the bulge $M_{\text {bulge }}$, since in general stars should dominate the gravitational potential on the scale of the bulge.

\subsection{Dynamical masses of the bulges}

Dynamical masses for the bulges are estimated as $M_{\mathrm{dyn}}=5 r_{e} \sigma^{2} / G$, where $r_{e}$ is the effective radius and $\sigma$ is the velocity dispersion of the bulge (Paper I). Here we use a prefactor of 5 to be consistent with Cappellari et al. (2006) and Sani et al. (2011). Dynamical masses for the sample of Gültekin et al. are from Häring \& Rix (2004). For the pseudobulges from Kormendv et al. (2011), we use effective radii from Sani et al. (2011). We also include 6 additional pseudobulges identified by (Sani et al. 2011, Figure 1 b). For the galaxies with stellar velocity dispersion measurements, the average difference between the predicted $\mathrm{BH}$ masses (based on the best fitting relation of Gültekin et al.'s sample and estimated dynamical masses) and observed values is $\left\langle\log \left(M_{\text {predict }} / M_{\mathrm{BH}}\right)\right\rangle=$ $0.79 \pm 0.09$. This offset is significantly larger than any reasonable formal uncertainty in the virial $\mathrm{BH}$ masses $(0.4-0.48$ dex $)$. Furthermore, even after correcting for stellar populations, our sample follows a steeper relation between $M_{\mathrm{BH}}$ and $M_{\text {bulge }}$ than the inactive ellipticals taken alone (Table 1). This is consistent with our observed Faber-Jackson relation in Paper I (Figure 9), in that at a fixed bulge magnitude, pesudobulges have smaller stellar velocity dispersions.

When a dark matter halo is included, the prefactor in the formula for dynamical mass decreases by 12\% (Cappellari et al. 2006). Furthermore, Tavlor et al. (2010) show that the normalization of dynamical mass formula for bulges with Sérsic index $n=2$ (as is the case for our sample of predominantly pseudobulges) is be larger by $\sim 40 \%$. Combining the above two effects would increase our estimated dynamical mass by $\sim 28 \%$. Considering the large uncertainty in the virial $\mathrm{BH}$ masses (a factor of 3), this is unlikely to change our results. Furthermore, if we were to boost the dynamical masses of the galaxies in our sample, the difference between our sample and Gültekin et al.'s sample would be larger.

Interestingly, the galaxies without extended disks show the largest offset (red triangles in Figure 1). They are also the largest outliers in the Faber-Jackson relation in Paper I. Rather than scaling like pseudobulges, these galaxies scale like spheroidals in the fundamental plane. We have no complete explanation for why these galaxies are the largest outliers, but it is intriguing ${ }^{5}$.

Finally, we look at pseudobulges (both active and inactive) and use the Kendall's $\tau$ rank correlation coefficient to test whether pseudobulge luminosity correlates with BH mass. The Kormendy et al. (2011) sample of pseudobulges taken alone has $\tau=0.44$ and $P=0.1$. There is no significant $M_{\mathrm{BH}}-M_{\text {bulge }}$ correlation among these galaxies. Including our sample yields $\tau=0.13$ and $P=0.03$. In this case there is marginal evidence for a very weak correlation. Thus we support the conclusion of Kormendy et al. (2011) that pseudobulges show no correlation between $\mathrm{BH}$ mass and bulge mass.

4. DISCUSSION AND CONCLUSION
We investigate the $M_{\mathrm{BH}}-L_{\text {bulge }}$ and $M_{\mathrm{BH}}-M_{\text {bulge }}$ relations focused on $10^{5}<M_{\mathrm{BH}} / M_{\odot}<10^{6}$. Both the $M_{\mathrm{BH}}-L_{\text {bulge }}$ and $M_{\mathrm{BH}}-M_{\text {bulge }}$ relations present the same conclusion that these galaxies appear to have smaller $\mathrm{BH}$ masses at a given bulge mass than more massive, bulge-dominated systems. Here we discuss the possible origins of this difference. It cannot be easily explained by errors in the $\mathrm{BH}$ mass scale. We would have to boost the $\mathrm{BH}$ masses by $\sim 1$ dex, which we consider unlikely given that the sample appears to obey an $M_{\mathrm{BH}}-\sigma_{*}$ relation (Barth et al. 2005; Greene \& Ho 2006; Xiao et al. 2011). Likewise, even correcting for young stellar populations in these bulges, we still find that our BHs are not consistent with the low mass extrapolation of the $M_{\mathrm{BH}}-L_{\text {bulge }}$ relation for more massive BHs (consistent with $\mathrm{Hu}$ 2008; Greene et al. 2008; Gadotti \& Kauffmann 2009; Greene et al. 2010).

As justified in Paper I, most of our galaxies are consistent with the properties of pseudobulges (e.g., Kormendy \& Kennicutt 2004; Fisher \& Drory 2010). We believe that pseudobulges are built by secular processes driven by disk instabilites. If the violent merging that builds classical bulges also establishes $\mathrm{BH}$-bulge scaling relations, we would expect different (or nonexistent) scalings for pseudobulges. We confirm the conclusion of Kormendy et al. (2011) that there is effectively no correlation between BH mass and pseudobulge luminosity based on our much larger sample. Furthermore, we extend this conclusion to bulge dynamical mass. This finding suggests that indeed, pseudobulges have quite different properties compared with classical bulges over a wide dynamic range, from $\sim 10^{5} M_{\odot}$ to $\sim 10^{8} M_{\odot}$ BHs. However, our sample is different from the sample of Kormendy et al. in that there is no systematic offset between the Kormendy et al. and the Gültekin et al. sample. Thus we see additional tentative evidence for larger systematic differences as a function of mass.

Taking our sample alone, the host galaxies have a wide range of bulge magnitudes over a very limited range in $\mathrm{BH}$ mass. In other words, the $M_{\mathrm{BH}}-L_{\text {bulge }}$ relation flattens out at the low-mass end. This seems to be consistent with the results from simulations of Volonteri \& Natarajan (2009) for massive BH seed models. If $\mathrm{BH}$ seeds can be as massive as $\sim 10^{5} M_{\odot}$, the low-mass BHs in our sample may be remnants of $\mathrm{BH}$ seeds that have not evolved much since they were formed. Consistent with our previous claims, we suggest that secular processes are relatively inefficient at feeding central BHs. Thus bulges that are built secularly maintain a low $\mathrm{BH}$ mass independent of the growth of their surrounding bulge.

\section{ACKNOWLEDGEMENTS}

Y.-F.J thanks Chien Peng for help in using GALFIT and Minjin Kim for helpful discussions on fitting the images. We also thank the anonymous referee for valuable comments to improve the manuscript. This work was supported by the Carnegie Institution for Science and by NASA grant HST-GO-11130.01 from the Space Telescope Science Institute, which is operated by AURA, Inc., under NASA contract NAS5-26555. 
${ }^{5}$ We have also divided our sample into galaxies with and without a bar. They do not show any significant difference.

\section{REFERENCES}

Barth, A. J., Sarzi, M., Rix, H.-W., Ho, L. C., Filippenko, A. V., \& Sargent, W. L. W. 2001, ApJ, 555, 685

Barth, A. J., Greene, J. E., \& Ho, L. C. 2005, ApJ, 619, L151

Bentz, M. C., Peterson, B. M., Pogge, R. W., Vestergaard, M., \& Onken, C. A. 2006, ApJ, 644, 133

Bentz, M. C., Peterson, B. M., Pogge, R. W., \& Vestergaard, M. 2009a, ApJ, 694, L166

Bentz, M. C., Peterson, B. M., Netzer, H., Pogge, R. W., \& Vestergaard, M. 2009b, ApJ, 697, 160

Blandford, R. D., \& McKee, C. F. 1982, ApJ, 255, 419

Cappellari, M., et al. 2006, MNRAS, 366, 1126

Ciotti, L., \& Ostriker, J. P. 2007, ApJ, 665, 1038

Collin, S., Kawaguchi, T., Peterson, B. M., \& Vestergaard, M. 2006, A\&A, 456, 75

Faber, S. M., \& Jackson, R. E. 1976, ApJ, 204, 668

Ferrarese, L., Pogge, R. W., Peterson, B. M., Merritt, D., Wandel, A., \& Joseph, C. L. 2001, ApJ, 555, L79

Fisher, D. B., \& Drory, N. 2010, ApJ, 716, 942

Gadotti, D. A. 2009, MNRAS, 393, 1531

Gadotti, D. A., \& Kauffmann, G. 2009, MNRAS, 399, 621

Gebhardt, K. 2004, in Carnegie Observatories Astrophysics Series, Vol. 1: Coevolution of Black Holes and Galaxies, ed. L. C. Ho (Cambridge: Cambridge Univ. Press), 248

Gebhardt, K., et al. 2000, ApJ, 543, L5

Greene, J. E., \& Ho, L. C. 2004, ApJ, 610, 722

Greene, J. E., \& Ho, L. C. 2006, ApJ, 641, L21

Greene, J. E., \& Ho, L. C. 2007, ApJ, 670, 92

Greene, J. E., \& Ho, L. C. 2009, PASP, 121, 1167

Greene, J. E., Ho, L. C., \& Barth, A. J. 2008, ApJ, 688, 159

Greene, J. E., et al. 2010, ApJ, 721, 26

Gültekin, K., et al. 2009, ApJ, 698, 198

Häring, N., \& Rix, H.-W. 2004, ApJ, 604, L89

Herrnstein, J. R., Moran, J. M., Greenhill, L. J., \& Trotter, A. S. 2005, ApJ, 629, 719

Hu, J. 2008, MNRAS, 386, 2242

Hu, J. 2009, arXiv:0908.2028

Jiang, Y.-F., Greene, J. E., Ho, L. C., Xiao, T., Barth, A. J. 2011, ApJ, accepted (Paper I)
Kaspi, S., Smith, P. S., Netzer, H., Maoz, D., Jannuzi, B. T., \& Giveon, U. 2000, ApJ, 533, 631

Kormendy, J., Bender, R., \& Cornell, M. E. 2011, Nature, 469, 374

Kormendy, J., \& Illingworth, G. 1983, ApJ, 265, 632

Kormendy, J., \& Kennicutt, R. C., Jr. 2004, ARA\&A, 42, 603

Kormendy, J., Fisher, D. B., Cornell, M. E., \& Bender, R. 2009, ApJS, 182, 216

Krist, J. 1995, Astronomical Data Analysis Software and Systems IV, 77, 349

Kuo, C. Y., et al. 2011, ApJ, 727, 20

Magorrian, J., et al. 1998, AJ, 115, 2285

Marconi, A., \& Hunt, L. K. 2003, ApJ, 589, L21

Nelson, C. H., Green, R. F., Bower, G., Gebhardt, K., \& Weistrop, D. 2004, ApJ, 615, 65

Onken, C. A., Ferrarese, L., Merritt, D., Peterson, B. M., Pogge,

R. W., Vestergaard, M., \& Wandel, A. 2004, ApJ, 615, 645

Peng, C. Y., Ho, L. C., Impey, C. D., \& Rix, H.-W. 2002, AJ, 124, 266

Peng, C. Y. 2007, ApJ, 671, 1098

Peng, C. Y., Ho, L. C., Impey, C. D., \& Rix, H.-W. 2010, AJ, 139, 2097

Sani, E., Marconi, A., Hunt, L. K., \& Risaliti, G. 2011, MNRAS, 443

Sérsic, J. L. 1968, Atlas de Galaxias Australes (Córdoba: Obs. Astron., Univ. Nac. Córdoba)

Shen, Y., Greene, J. E., Strauss, M. A., Richards, G. T., \& Schneider, D. P. 2008, ApJ, 680, 169

Taylor, E. N., Franx, M., Brinchmann, J., van der Wel, A., \& van Dokkum, P. G. 2010, ApJ, 722, 1

Tremaine, S., et al. 2002, ApJ, 574, 740

Volonteri, M., \& Natarajan, P. 2009, MNRAS, 400, 1911

Woo, J.-H., et al. 2010, ApJ, 716, 269

Xiao, T., Barth, A. J., Greene, J. E., Ho, L. C., Bentz, M. C., Ludwig, R. R., \& Jiang, Y. 2011, arXiv:1106.6232 